\title{
Career \\ coaching for individuals
}

Helen Sang

The engine of research in biological studies is the PhD student/postdoctoral scientist body. It is very challenging for an individual to move from being a postdoc to becoming a principal investigator ( $\mathrm{PI})$, and, once a lecturer, to progress up the academic career ladder. The opportunity to step outside day-to-day work and line management to reflect on goals and progress with a coach can make a huge difference.

This recipe explains the steps used at the Roslin Institute to get the career coaching scheme up and running. Be aware that it can be expensive when done with external providers - the cost can be as much as $£_{300}$ per person.

\section{Ingredients}

- Money (so that the department can pay for the coaching).

- An individual who wants to develop themselves.

- Aclear-headed experienced independent person to make the selection.

- A supportive environment including a supportive line manager - to facilitate the outcomes of the coaching experience.

- A qualified coach.

\section{Method}

1. Find the people who will put most into coaching, and benefit most from it. Advertise the opportunity for coaching. In their applications, individuals must describe their reasons for wanting coaching. Have the selection done by an independent person, based on the applicants' reasons.

2. Set up the beginning and end parameters of the coaching process.

3. Coachees must be allowed time set aside for coaching. Line managers should be aware of the coaching process and encourage coachees to implement their plans.

4. Individual coaches work with coachees to identify their personal challenges in career aims and ambitions.

5. Individuals develop action plans, seek additional training opportunities, and in turn give better support to workplace colleagues.

6. Close the organisational learning loop. Coaches can give feedback to the organisation on how in-house career development support could be improved. Remember that individuals' experiences must be kept confidential.

131 EqualBITE Career coaching for individuals 


\section{Additional notes}

This recipe focuses on working with external coaches and this is always a potential option for departments and line managers to consider. There are also a number of trained internal coaches in the University, most of whom are based in the Institute for Academic Development, the Business School and University Human Resources (HR) Services.

Working with an internal coach can be more cost-effective (this is usually part of someone's role and is not normally a service with an additional fee). Having a coach who is familiar with the University culture can be a benefit for some clients.

A research fellow in the Roslin Institute participated in a coaching programme from the Institute for Academic Development. Here are some of her reflections on the experience:

$$
\text { ience: }
$$

I started coaching without having a clue what coaching was about. Now, some years later, I know that this was one of the best presents the University of Edinburgh has given me. I am really grateful to my coach who helped me to understand initially what I wanted work-wise and then how to get there. In a very short time I became more effective, efficient and confident.

With coaching I managed to deal in a productive way with all the obstacles I came across. With reflection and good guiding I overcame the confusion and stress I had regarding my workplace and I became more motivated.
My coach provided the voice of logic when I could not see things clearly, and helped me understand the culturenot always straightforward since I come from a different background. She supported me through difficult personal times of loss, and helped me to create a good work-life balance during and after my pregnancy.

I met almost all my coaching targets, and I highly recommend the process to anyone who wants to advance their career and have a balanced life. 